\title{
Continuous Positive Airway Pressure Treatment and Anxiety in Adults with Coronary Artery Disease and Nonsleepy Obstructive Sleep Apnea in the RICCADSA Trial
}

\section{Yeliz Celik $^{1}$, Erik Thunström ${ }^{2}$, Patrick J Strollo Jr ${ }^{3}$, Yüksel Peker ${ }^{1,2,3,4}$}

${ }^{1}$ Koc University Research Center for Translational Medicine (KUTTAM), Istanbul, Turkey;

${ }^{2}$ Dept. of Molecular and Clinical Medicine/Cardiology, Sahlgrenska Academy, University of

Gothenburg, Sweden; ${ }^{3}$ Dept of Clinical Sciences, Respiratory Medicine and Allergology, Faculty of Medicine, Lund University, Lund, Sweden; ${ }^{4}$ Division of Pulmonary, Allergy, and Critical Care Medicine, University of Pittsburgh School of Medicine, Pittsburgh, PA, USA.

ORCID IDs: 0000-0002-4041-4529 (Y.C.); 0000-0002-9134-2107 (E.T.); 0000-0002-20659641 (P.J.S.); 0000-0001-9067-6538 (Y.P.).

Corresponding author: Yüksel Peker, $\mathrm{MD}, \mathrm{PhD}$

Dept of Pulmonary Medicine, Koc University, School of Medicine

Koc University Hospital, Davutpasa cad, No 4

TR-34010 Zeytinburnu, Istanbul, Turkey.

Email: yuksel.peker@lungall.gu.se

Authors' contributions: YP designed the study. YP and ET performed the patient recruitment and clinical follow-ups. YC and YP performed the statistical analysis. All authors 
interpreted the data, prepared the manuscript, and drafted the article. YP obtained study funding, and takes full responsibility for the work as a whole, including the study design, access to data, and the decision to submit and publish the manuscript. All authors approved this manuscript in its final form.

Funding support: This study was supported by grants from the Swedish Research Council (521-2011-537 and 521-2013-3439); the Swedish Heart-Lung Foundation (20080592, 20090708 and 20100664); the "Agreement concerning research and education of doctors" of Västra Götalandsregionen (ALFGBG-11538 and ALFGBG-150801), Research fund at Skaraborg Hospital (VGSKAS-4731, VGSKAS-5908, VGSKAS-9134, VGSKAS-14781, VGSKAS-40271 and VGSKAS-116431); Skaraborg Research and Development Council (VGFOUSKB-46371); the Heart Foundation of Kärnsjukhuset; ResMed Foundation; and ResMed Ltd. ResMed Sweden provided some of the sleep recording devices and technical support. None of the funders had any direct influence on the design of the study, the analysis of the data, the data collection, drafting of the manuscript, or the decision to publish.

Running Head : CPAP and Anxiety in CAD with Nonsleepy OSA

Descriptor: 15.09 Sleep Disordered Breathing: Outcomes

Word count without Abstract, References or Tables: 3784 


\section{ABSTRACT}

Background: Anxiety and obstructive sleep apnea (OSA) coexist among adults with coronary artery disease $(\mathrm{CAD})$ following revascularization. Continuous positive airway pressure (CPAP) is the first line treatment of OSA patients with daytime sleepiness. The current study evaluated the effect of CPAP on anxiety in CAD patients with nonsleepy OSA. Methods: Two hundred forty-four revascularized CAD patients with nonsleepy OSA (apneahypopnea index $\geq 15 / \mathrm{h}$, Epworth Sleepiness Scale score $<10$ ) were randomly assigned to CPAP or no-CPAP between 2005 and 2010. Zung Self-rating Anxiety Scale (SAS) was administered at baseline, and after 3 and 12 months with higher scores suggesting more anxiety.

Results: A total of 208 patients with complete SAS scores at baseline and 12-month followup were included (CPAP, $n=103$; no-CPAP, $\mathrm{n}=105$ ). In the intention-to-treat analysis, CPAP had no significant effect on the SAS scores. On-treatment analysis revealed a significant increase in the median of delta SAS score $(+3.75)$ after three months among the participants using the device 2.8 hours/day or more while there was a decline in the median of delta SAS score $(-1.25)$ in the non-adherent or no-CPAP group $(p=0.031)$. The increase in the SAS score $(+1.25)$ in the adherent group, and the decline (-1.25 points) in the non-adherent/noCPAP group remained significant after one year $(p=0.011)$. Baseline SAS score predicted non-adherence (adjusted odds ratio 1.11; 95\% confidence interval [CI] 1.04-1.18; $p=0.003$ ), and there was an association between the increase in the SAS scores and accumulated CPAP hours/day (standardized $\beta=0.144$ [95\% CI 0.005-0.695], $p=0.047$ ).

Conclusion: Our results suggest that anxiety should be considered in the management of CAD patients with nonsleepy OSA following revascularization.

Clinical Trial Registration: NCT00519597.

Word count: 267 
Keywords: Coronary Artery Disease, Obstructive Sleep Apnea, Anxiety, CPAP 


\section{INTRODUCTION}

Coronary artery disease (CAD) is a common disorder leading to enhanced morbidity and mortality worldwide (1). Although traditionally recognized cardiovascular risk factors play an important role on the pathogenesis of CAD, there is also data indicating that psychological factors should also be considered in this context (2). As also emphasized in a recent review, cardiac patients are more likely to report impaired sleep with reduced sleep duration and quality, and also, sleep interruptions due to nocturnal dyspnea or arrhythmias (3).

Anxiety is one of the prominent psychological symptoms with an estimated prevalence rate ranging from 20 to $50 \%$ among adults with $\mathrm{CAD}(4,5)$. Previous studies on anxiety have been generally performed among cardiac patients before and after mechanical revascularizations such as percutaneous coronary intervention (PCI) (6), and coronary artery bypass grafting (CABG) surgery (7-9). The results of these studies imply that preoperative anxiety aggravates complications experienced during early postoperative period including hemodynamic consequences, prolonged mechanical ventilation, higher postoperative pain, increased analgesic and anaesthetic requirements (10). Consequently, postoperative anxiety has also been related with a 4-year mortality among patients undergoing CABG and valve surgery (11).

Obstructive sleep apnea (OSA) is also a common disorder (12), and anxiety has been reported among $32 \%$ of OSA patients according to a recent systematic review and meta-analysis (13).

On the other hand, there have also been reports suggesting no association between OSA and anxiety symptoms $(14,15)$. Continuous positive airway pressure (CPAP), which is the first line treatment of symptomatic OSA patients, has been shown to improve health related quality of life (16) and comorbid depression $(17,18)$ but not anxiety $(19)$. In the secondary analysis of the Sleep Apnea Cardiovascular Endpoints (SAVE) trial, that involved 2410 
patients with cardiovascular and/or cerebrovascular disease and co-existing OSA, there were no significant changes in anxiety symptoms following CPAP treatment (20). No reports regarding the association between OSA treatment and anxiety are yet available from other recently completed or ongoing RCTs evaluating the cardiovascular effects of the OSA treatment as primary outcomes (21-25).

The RICCADSA (Randomized Intervention with CPAP in CAD and OSA) trial primarily addressed the effects of CPAP on the composite of repeat revascularization, myocardial infarction, stroke, and cardiovascular mortality in revascularized patients with CAD and nonsleepy OSA (26). In the current work, we evaluated the impact of CPAP on anxiety in the same cohort as one of the secondary outcomes of the RICCADSA trial.

\section{METHODS}

\section{Study Design and Participants}

The study design has been reported in detail elsewhere (27). In brief, all consecutive CAD patients undergoing PCI or CABG in the Skaraborg County of West Götaland, Sweden between 2005 and $2010(n=1,291)$ were invited to participate (Figure 1). The participants with nonsleepy OSA (apnea-hypopnea index $[\mathrm{AHI}]>15 / \mathrm{h}$ ) based on home sleep apnea testing [HSAT], and Epworth Sleepiness Scale [ESS] scores less than 10 points), were included in the randomized controlled trial (RCT) arm for the main protocol. For the purpose of the current study, the patients who completed Zung Self-rating Anxiety Scale (SAS) at baseline and follow-ups were included. The main study was completed in May 2013. The study protocol was approved by the Ethics Committee of the Medical Faculty of the University of Gothenburg (approval no. 207-05; 09.13.2005; amendment T744-10; 11.26.2010; amendment T512-11;06.16.2011), and written informed consent was provided by all patients. The trial was registered (FoU i Sverige-Research and Development in 
Sweden; researchweb.org; VGSKAS-4731; 04.29.2005; and www. clinicaltrials.gov; NCT00519597).

\section{Home Sleep Apnea Testing}

As previously described in detail (27), the Embletta Portable Digital System device was used for the HSAT recordings. The system contains a detector for nasal pressure, a finger pulse oximeter to detect both oxyhemoglobin saturation and heart rate as well as two respiratory belts for body position and thoraco-abdominal movements. Since the study protocol was established in 2005, the Chicago criteria were applied for apnea and hypopnea definitions (28); i.e., at least $90 \%$ cessation of airflow was recognised as an apnea, and at least $50 \%$ decrease in nasal pressure amplitude and/or thoracoabdominal movement for 10 seconds or longer, irrespective of an oxyhemoglobin desaturation was scored as a hypopnea. The total number of oxyhemoglobin desaturations of at least $4 \%$ from the immediately preceding baseline, was also measured, and oxygen desaturation index (ODI) was calculated.

\section{Epworth Sleepiness Scale}

ESS, a self-administered questionnaire with eight questions to address the possibility of dozing off while performing eight different activites in the past month, was used to determine excessive daytime sleepiness (29). The participants with an ESS score less than 10 points were categorized as the nonsleepy phenotype.

\section{Zung Self-rated Anxiety Scale}

Participants were requested to answer the Zung SAS at baseline and 3 and 12 months after randomization. The Zung SAS is a likert scale which compromises 20 items to measure physiological and psychological symptoms (30). Each item was rated on a 4-point scale 
ranging from 1 (none, or a little of the time) to 4 (most, or all of the time), and the raw scores (range 20-80) were converted into index scores (range 25-100) by multiplying 1.25 . The SAS items include both affective and somatic symptoms; 15 items defining a negative experience such as "I feel afraid for no reason at all", and 5 items expressing a positive experience such as "I can breathe in and out easily". The positive items were recoded reversely (30). Individuals with a SAS score of at least 45 points were defined as having a clinically significant anxiety, and higher scores indicate more anxiety (31). The questionnaire is provided in the online supplement (eTable 1). As item 19 ("I fall asleep easily and get a good night's rest") as well as item 20 ("I have nightmares") may overlap symptoms related to OSA, a separate analysis was performed by excluding these questions from the total scores. All responses were recorded in the local database by a research nurse blinded to the group allocation and clinical data.

\section{Comorbidities}

Information regarding anthropometrics, smoking habits, comorbidities and use of medications were obtained at baseline. Obesity was defined as a body mass index (BMI) of $30 \mathrm{~kg} / \mathrm{m}^{2}$ or greater (32).

\section{Group Assignment, Randomization, Interventions, and Follow-Up}

As illustrated in Figure 1, the group assignment of the RCT arm was based on the HSAT results and the ESS scores. Nonsleepy OSA patients, who were randomly allocated to CPAP were provided with an automatic (self-titrating) CPAP device (S8 or S9; ResMed) with a nasal or full-face mask and humidifier. As previously described elsewhere (27), the CPAP group were contacted by telephone after 1 week, and both groups were followed after 1 month, 3 months, 6 months, 1 year, and then yearly until the end of the main study. 


\section{Adherence to CPAP}

Participants allocated to CPAP brought the machines to the clinic at each follow-up, and CPAP use (hours/night) were downloaded from the device and entered into the database. Technical adjustments were done according to the clinical routines by the sleep medicine unit staff. Participants who stopped using the their devices were followed as part of the ITT population (27). Accumulated CPAP usage (CPAP hours per day adjusted for percentage of CPAP days per 3 months and 12 months, respectively) was documented for each participant allocated to CPAP. Adherence was defined as CPAP usage at least 4 hours/day and 70\% CPAP days/period (33), corresponding 2.8 hours/day/all days.

\section{Outcomes and the sample size}

The major outcome of the current study, which was one of the secondary endpoints of the RICCADSA trial, was absolute change from baseline in the SAS scores at 3 and 12 months, respectively. The sample size estimation for the RICCADSA trial was based on the calculations for the primary composite endpoints (i.e, long-term cardiovascular adverse events) (26).

\section{Statistical analysis}

For descriptive statistics, the data are presented as medians and boundaries of interquartile ranges (continuous variables), and as percentages (categorical variables). Mann-Whitney U test was used to compare the difference between two study groups for continous variables. Chi-Square test (or, when appropriate, Fisher's exact test) was conducted to compare categorical variables. Primary analysis was conducted in intention-to-treat population. Additional analyzes were conducted to compare the anxiety levels of participants allocated to no-CPAP and nonadherent OSA with participants who were adherent to CPAP. Pearson 
correlation coefficient was calculated between delta SAS scores and accumulated CPAP hours/day at 12-month follow-up. To be in line with the recent guidelines(34), the covariates for multivariate analyses were selected carefully. Age, sex, type of revascularization, comorbidities, ESS scores, Zung Self-rating Depression Scale scores, and CPAP usage were entered into the model to address the correlates of delta SAS scores. Baseline SAS scores were not included into the model due to multicollinearity. The tests were performed as twosided and $p$ value less than 0.05 was considered as statistically significant. The data was analysed by using Statistical Package for Social Sciences, version 26.0 for Windows system (SPSS Inc.).

\section{RESULTS}

\section{Clinical Characteristics of the Study Cohort}

As shown in Figure 1, 244 revascularized CAD patients with nonsleepy OSA was included in the RCT arm of the main trial. After excluding 34 patients who did not respond to the Zung SAS at baseline and/or at 12 month follow-up, 208 (mean age $65.9+8.1$ years; $83.7 \%$ men) remained for the current study (103 allocated to CPAP, 105 to no-CPAP). As shown in Table 1, diabetes mellitus was more common among patients allocated to CPAP whereas the distribution of the SAS scores and other baseline characteristics were similar. In all, 37 patients $(17.8 \%)$ presented SAS scores $\geq 45$ with a slightly higher occurrence in the no-CPAP group (Table 1). None of the patients was on hypnotics or anxiolytic drugs as continuous medication. As shown in online supplement eTable 2, baseline characteristics of participants with SAS scores $\geq 45$ did not differ significantly between the CPAP $v s$ no-CPAP groups except depressive mood which was more common among the participants allocated to the noCPAP at baseline. Within the CPAP group with anxiety at baseline $(n=14)$, seven returned the 
device within one year, and four out of remaining seven were using the device less than 2.8 hours/day at the 12-month follow-up.

As illustrated in online supplement eFigure 1, 34.0\% in the CPAP group, and $38.1 \%$ in the no-CPAP group had answered as "little of the time" or "some of the time" to the item 19 (I fall asleep easily and get a good night's rest), and 3.9\% in the CPAP group, and $4.8 \%$ in the no-CPAP group had answered as "most of the time" or "good part of the time" to the item 20 "I have nightmares". Items 5 and 17 were the other questions that were rated more frequently in the entire population (39.0\% and $41.3 \%$, respectively) with no between-group differences.

\section{CPAP Usage}

In the CPAP group, 31 (30.1\%) stopped using the device within 12 months. As shown in online supplement eTable 3, the patients who stopped using the device within one year had similar characteristics at baseline compared with the ones remaining on CPAP. The main reasons for returning the CPAP device were stated as "discomfort" (51.6\%), "anxiety" (25.8\%), insomnia (16.1\%), and "others (the partner dislikes the device, etc.)" (6.5\%). In the no-CPAP group ( $n=103$ ), five patients had crossed-over to the CPAP arm within the followup period (Figure 1).

\section{Outcomes}

\section{Intention-to-treat population}

The distribution of the SAS scores were similar at baseline and at follow-ups (Table 2). As illustrated in Figure 2A, there was a non-significant decrease in change from baseline after 3 months in both groups, and this was more prominent after 12 months, especially in the noCPAP group. Among patients with no anxiety at baseline $(n=171), 11$ in the CPAP group, and 
7 in the no-CPAP group demonstrated SAS score $\geqslant 45$ at 12 month-follow-up (n.s.). No changes were observed regarding prescription of anti-depressive medications during the follow-up period, and none of the patients were hospitalized due to psychiatric reasons.

As illustrated in Figure 3, there was a weak but significant association between accumulated CPAP hours/day and change from baseline on the SAS scores at 12-month follow-up ( $\mathrm{r}=0.14$, $p=0.042$ ). Among the patients randomized to CPAP, the baseline SAS scores predicted nonadherence (adjusted odds ratio $1.11 ; 95 \%$ confidence interval $[\mathrm{CI}] 1.04-1.18 ; p=0.003$ ) (Table 3). In the entire study population, accumulated CPAP hours/day remained as the only significant variable associated with the delta SAS scores at 12-month follow-up in the multivariate analysis (Table 4).

\section{On-treatment population}

As shown in Table 2, the on-treatment analysis revealed that the median SAS score at baseline was significantly higher in the non-adherent/no-CPAP group compared to the participants who were using CPAP $\geq 2.8$ hours/day/all days at the 12-month follow-up. As

illustrated in Figure 2B, the patients in the non-adherent/no CPAP group rated lower anxiety scores at follow-ups compared to baseline while there was an increased anxiety in the adherent group, which was more prominent after 3 months. Analysis showed that the median delta score $(+3.75)$ in the adherent group was significantly higher than the median delta score in the non-adherent/no-CPAP group $(-1.25)$ after 3 months $(p=0.031)$. The difference in the median SAS scores remained significant after 12 months follow-up $(+1.25$, and $-1.25, p=$ 0.011 , respectively).

\section{DISCUSSION}


The present study showed no significant difference in anxiety levels among the CAD patients with nonsleepy OSA, who were randomized to CPAP or no-CPAP in intention-to-treat population. On-treatment analysis revealed a significant increase in the anxiety levels after three months among the individuals using the device at least 2.8 hours/day. The magnitude of the increase in the anxiety level reduced but remained significant at 12-month follow-up.

To the best of our knowledge, the present study is the first RCT evaluating the effect of CPAP treatment on anxiety in a CAD population with concomitant OSA. In the SAVE trial, that involved a larger number of participants with $\mathrm{CAD}$ and cerebrovascular disease, CPAP treatment had a neutral impact on anxiety scores based on the Hospital Anxiety and Depression Scale-Anxiety (HADS-A) (20). In the detailed analysis of these results, there was a significant decrease in anxiety symptoms after six months of CPAP treatment among the cases with high scores at baseline, but not at the subsequent assessments. It was argued in an editorial that the lack of improvement in the anxiety scores after CPAP treatment could be due to the low occurrence of anxiety cases at baseline (19). However, the magnitude of the reduction in the scores among the cases did not differ between the CPAP and usual care groups, indicating a poor efficacy of OSA treatment. Indeed, our results suggest that CPAP treatment is associated with an increase rather than a decrease of the anxiety symptoms in CAD patients who are nonsleepy. Given that a large number of the SAVE cases had mild sleepiness (ESS 10-15) at baseline, the improvement in the ESS following CPAP treatment may have contributed to the reduction observed in the anxiety scores at 6 months follow-up in that trial.

Anxiety is defined as a state of uncertainty about future threats, which is accompanied by concern, stress, negative emotions and sense of insecurity (35). It is associated with several cardiovascular pathophysiological processes such as cardiac arrhythmias and increased 
autonomic nervous activity (11). Previous studies have demonstrated that the "heartfocused" anxiety symptoms including, fear, tension, high stress, headache, sweating, muscle spasms and palpitations are common in cardiac populations (36). Anxiety as a state can be a transient reaction directly associated to a specific health-related intervention (37). This transient anxiety may expand to a persistent state, namely, trait anxiety. This type of anxiety has been reported in CAD patients who were hospitalized for cardiovascular events during a four-year period after CABG (11). Hence, comorbid anxiety appears to be a risk factor for $\mathrm{CAD}$, and may play crucial roles on the onset, development, management and response to treatment in these high-risk populations. Previous studies have been focused on the incidence and course of the anxiety among CAD cohorts before and after the revascularization procedures $(38,39)$. However, neither of them has considered OSA in the evaluation of anxiety in revascularized CAD patients. In the present study, not only the traditionally defined OSA in sleep clinic cohorts, but also the nonsleepy phenotype as well as response to CPAP treatment has been examined.

Other studies conducted in sleep clinic cohorts have mainly addressed the relationship between OSA severity and anxiety symptoms $(14,15,40,41)$, and a recent meta-analysis found no significant association (20). It is noteworthy that there was no association between type of questionnaire and the anxiety prevalence in the subgroup of studies included in that meta-analysis (20).

In the current study, approximately one fifth of the cohort was categorized as having anxiety based on the suggested SAS score threshold $(\geq 45)$ with no significant difference between the CPAP and no-CPAP groups. Compared to the previous occurrence rates ranging from 20 to $50 \%$ among adults with $\mathrm{CAD}(4,5)$, the prevalence of anxiety in our cohort was at the lower edge. One possible explanation could be the demographic, cultural and clinical differences 
between the studied cohorts as well as the scales used in evaluating anxiety. Most of the studies had reported results based on the HADS-A (20); however, no significant difference has been found between HADS-A and SAS in classifying the severity of anxiety (42). In the SAVE trial, the prevalence rates were similar in both CPAP and usual care groups $(\% 14.4 v s$ $\% 17.0)$ at the end of the follow-up (20).

We observed no significant change in the SAS scores after 12 months in the entire nonsleepy OSA population. A possible argument for this finding could be the fact that the overall scores were below the threshold among $80 \%$ of the population, i.e, the majority of the CAD patients had no or mild symptoms of anxiety at baseline. However, in the subgroup of participants with the SAS scores over the cut-off value, there was an improvement in the state anxiety among the participants allocated to no-CPAP whereas the severity of anxiety remained unchanged among the cases randomized to CPAP. Though the sample size of clinically anxious patients (SAS score $\geq 45$ ) allocated to CPAP at baseline was small, the most of them had returned the device within one year. Moreover, there was a significant association between the baseline SAS scores and non-adherence among the CPAP group, which may imply that subclinical anxiety should be considered in the management of CPAP treatment of CAD patients with the nonsleepy OSA.

Remarkably, there was a significant increase in the delta SAS scores already after 3 months in the whole population among the patients using the device at least 2.8 hours/night, while there was a decline in the non-adherent/no-CPAP group. The significant association between increase in the anxiety scores and CPAP use in the multivariate analysis may suggest that CPAP treatment may worsen symptoms of anxiety among CAD patients with nonsleepy OSA following PCI or CABG. This increase might be temporary given that the magnitude of the 
change was reduced at the 12-month follow-up. It is also probable that nonsleepy cardiac patients develop a higher degree of anxiety when they are asked to use CPAP in order to avoid adverse cardiovascular effects of OSA.

Of note, the results of the current study are not applicable to CAD patients with sleepy OSA phenotype. Given that sleepy patients are more prone to be adherent to CPAP therapy compared to the nonsleepy individuals (43), it may be argued that the reduction in excessive daytime sleepiness after CPAP treatment counteracts the anxiety symptoms in the sleepy OSA phenotype. Further analysis of the RICCADSA cohort comparing anxiety symptoms in sleepy vs nonsleepy OSA patients and adherence to CPAP treatment is in progress, and will be reported in future work.

\section{Limitations}

This study has a number of limitations that need to be acknowledged. First, the power estimate for the RCT population was done for the primary outcome (composite of repeat revascularization, myocardial infarction, stroke, and cardiovascular mortality), and not for the outcomes addressed in the current study. Second, there is yet no consensus regarding how much change in SAS scores should be considered as minimum clinically important difference (MCID) in CAD patients with concomitant OSA following CPAP treatment. Though 2 points of change in HADS-A scores was suggested as MCID (44), the studies summarized in the recent meta-analysis failed to show such a significant effect of CPAP treatment (20). In the current study, we found a 3.75 points of increase in the median of delta anxiety scores after 3 months among the nonsleepy OSA patients using the device at least 2.8 hours/night. Third, CPAP usage data were downloaded at each follow-up period for the main trial after 3 months, 6 months, 12 months, and yearly up to 6 years, so the downloaded CPAP usage data for the 
month prior to completing the questionnaire were not recorded separately. Thus, the patient's CPAP use during the last month prior to the 12-month follow-up could possibly better reflect the association between CPAP usage and anxiety state. Fourth, results of the on-treatment analysis should be interpreted cautiously, because no randomization at baseline to "adherent" versus "nonadherent" is possible. Given that device usage and non-usage is patient-driven, a self-selection bias can therefore not be excluded. Finally, the age distribution of the study cohort is not typical for the OSA population, and our results are, therefore, not generalizable to younger sleep clinic cohorts with symptomatic OSA. Nevertheless, the secondary outcome analysis of the current study gives important insight into how anxiety level is affected by CPAP in revascularized patients with CAD and nonsleepy OSA.

\section{CONCLUSIONS}

CPAP prescription to nonsleepy CAD patients with OSA had neutral effect on anxiety levels in intention-to-treat population. However, on-treatment analysis revealed a significant increase in anxiety levels after three months among patients using the device at least 2.8 hours/day. The magnitude of the increase in the anxiety level reduced but remained significant as well at 12-month follow-up. Thus, it seems crucial to personalize the management of CAD patients to include early diagnosis and treatment of anxiety. This is particularly important among nonsleepy OSA phenotype in cardiac populations undergoing PCI or CABG, for whom CPAP treatment is considered in order to decrease the risk for adverse cardiovascular outcomes.

\section{Acknowledgment}

The authors gratefully acknowledge research nurse Alen Salkic for data entry of the Zung Self-Rating Anxiety Scale questionnaires. 


\section{Data sharing statement}

Individual participant data that underlie the results reported in this article can be obtained by contacting the corresponding author, yuksel.peker@lungall.gu.se.

\section{Disclosure Statements}

\section{Financial Disclosure}

YC and ET report no conflicts of interest. PS received institutional grants from PhilipsRespironics, ResMed, Inspire Medical Systems, National Football League PinMed, and advisory fees from ResMed, Emmi Solutions, Jazz Pharmaceuticals, Itamar Medical, Inspire Medical Systems, Seperation Design Group (all outside the current work). YP received institutional grants from ResMed for the current work.

\section{Non-financial Disclosure}

None.

\section{REFERENCES}

1. Nowbar AN, Gitto M, Howard JP, Francis DP, Al-Lamee R. Mortality From Ischemic Heart Disease: Analysis of Data From the World Health Organization and Coronary Artery Disease Risk Factors From NCD Risk Factor Collaboration. Circulation: Cardiovascular Quality and Outcomes 2019; 12: e005375.

2. Watkins LL, Koch GG, Sherwood A, Blumenthal JA, Davidson JR, O'Connor C, Sketch Jr MH. Association of anxiety and depression with all-cause mortality in individuals with coronary heart disease. Journal of the American Heart Association 2013; 2: e000068.

3. Spiesshoefer J, Linz D, Skobel E, Arzt M, Stadler S, Schoebel C, Fietze I, Penzel T, Sinha AM, Fox H, Oldenburg O, On Behalf Of The German Cardiac Society Working Group On Sleep Disordered Breathing Ag-Deutsche Gesellschaft Für Kardiologie Herz Und Kreislaufforschung EVO. Sleep - the yet underappreciated player in cardiovascular diseases: A clinical review from the German Cardiac Society Working Group on Sleep Disordered Breathing. Eur J Prev Cardiol 2019: 2047487319879526.

4. Thombs BD, Bass EB, Ford DE, Stewart KJ, Tsilidis KK, Patel U, Fauerbach JA, Bush DE, Ziegelstein RC. Prevalence of depression in survivors of acute myocardial infarction. $J$ Gen Intern Med 2006; 21: 30-38.

5. Musselman DL, Evans DL, Nemeroff CB. The relationship of depression to cardiovascular disease: epidemiology, biology, and treatment. Archives of general psychiatry 1998; 55: 580-592. 
6. Pedersen SS, Smith OR, De Vries J, Appels A, Denollet J. Course of anxiety symptoms over an 18month period in exhausted patients post percutaneous coronary intervention. Psychosom Med 2008; 70: 349-355.

7. Murphy BM, Elliott PC, Worcester MU, Higgins RO, Le Grande MR, Roberts SB, Goble AJ. Trajectories and predictors of anxiety and depression in women during the 12 months following an acute cardiac event. Br J Health Psychol 2008; 13: 135-153.

8. Duits AA, Duivenvoorden HJ, Boeke S, Taams MA, Mochtar B, Krauss XH, Passchier J, Erdman RA. The course of anxiety and depression in patients undergoing coronary artery bypass graft surgery. J Psychosom Res 1998; 45: 127-138.

9. Blumenthal JA, Lett HS, Babyak MA, White W, Smith PK, Mark DB, Jones R, Mathew JP, Newman MF. Depression as a risk factor for mortality after coronary artery bypass surgery. Lancet 2003; 362: 604-609.

10. Bailey L. Strategies for decreasing patient anxiety in the perioperative setting. Aorn $j$ 2010; 92: 445-457; quiz 458-460.

11. Szekely A, Balog P, Benko E, Breuer T, Szekely J, Kertai MD, Horkay F, Kopp MS, Thayer JF. Anxiety predicts mortality and morbidity after coronary artery and valve surgery--a 4-year follow-up study. Psychosom Med 2007; 69: 625-631.

12. Heinzer R, Vat S, Marques-Vidal P, Marti-Soler H, Andries D, Tobback N, Mooser V, Preisig M, Malhotra A, Waeber G, Vollenweider P, Tafti M, Haba-Rubio J. Prevalence of sleepdisordered breathing in the general population: the HypnoLaus study. Lancet Respir Med 2015; 3: 310-318.

13. Garbarino S, Bardwell WA, Guglielmi O, Chiorri C, Bonanni E, Magnavita N. Association of Anxiety and Depression in Obstructive Sleep Apnea Patients: A Systematic Review and MetaAnalysis. Behav Sleep Med 2020; 18: 35-57.

14. Sforza E, Saint Martin M, Barthelemy JC, Roche F. Mood disorders in healthy elderly with obstructive sleep apnea: a gender effect. Sleep medicine 2016; 19: 57-62.

15. Lehto SM, Sahlman J, Soini EJ, Gylling H, Vanninen E, Seppa J, Viinamaki H, Tuomilehto H. The association between anxiety and the degree of illness in mild obstructive sleep apnoea. Clin Respir J 2013; 7: 197-203.

16. Engleman HM, Martin SE, Kingshott RN, Mackay TW, Deary IJ, Douglas NJ. Randomised placebo controlled trial of daytime function after continuous positive airway pressure (CPAP) therapy for the sleep apnoea/hypopnoea syndrome. Thorax 1998; 53: 341-345.

17. Povitz M, Bolo CE, Heitman SJ, Tsai WH, Wang J, James MT. Effect of treatment of obstructive sleep apnea on depressive symptoms: systematic review and meta-analysis. PLoS Med 2014; 11: e1001762.

18. Balcan B, Thunstrom E, Strollo PJ, Jr., Peker Y. Continuous Positive Airway Pressure Treatment and Depression in Adults with Coronary Artery Disease and Nonsleepy Obstructive Sleep Apnea. A Secondary Analysis of the RICCADSA Trial. Annals of the American Thoracic Society 2019; 16: 62-70.

19. Marrone O. Mood After CPAP: Fewer Patients With Depression, but not Fewer With Anxiety. EClinicalMedicine 2019; 11: 9-10.

20. Zheng D, Xu Y, You S, Hackett ML, Woodman RJ, Li Q, Woodward M, Loffler KA, Rodgers A, Drager LF, Lorenzi-Filho G, Wang X, Quan WW, Tripathi M, Mediano O, Ou Q, Chen R, Liu Z, Zhang X, Luo Y, McArdle N, Mukherjee S, McEvoy RD, Anderson CS. Effects of continuous positive airway pressure on depression and anxiety symptoms in patients with obstructive sleep apnoea: results from the sleep apnoea cardiovascular Endpoint randomised trial and meta-analysis. EClinicalMedicine 2019; 11: 89-96.

21. Fox H, Hetzenecker A, Stadler S, Oldenburg O, Hamer OW, Zeman F, Bruch L, Seidel M, Buchner $\mathrm{S}$, Arzt M. Rationale and design of the randomised Treatment of sleep apnoea Early After Myocardial infarction with Adaptive Servo-Ventilation trial (TEAM-ASV I). Trials 2020; 21: 129. 
22. Sharma S, Fox H, Aguilar F, Mukhtar U, Willes L, Bozorgnia B, Bitter T, Oldenburg O. Auto positive airway pressure therapy reduces pulmonary pressures in adults admitted for acute heart failure with pulmonary hypertension and obstructive sleep apnea. The ASAP-HF Pilot Trial. Sleep 2019; 42.

23. Fox H, Bitter T, Sauzet O, Rudolph V, Oldenburg O. Automatic positive airway pressure for obstructive sleep apnea in heart failure with reduced ejection fraction. Clin Res Cardiol 2020.

24. Sánchez-de-la-Torre M, Sánchez-de-la-Torre A, Bertran S, Abad J, Duran-Cantolla J, Cabriada V, Mediano O, Masdeu MJ, Alonso ML, Masa JF, Barceló A, de la Peña M, Mayos M, Coloma R, Montserrat JM, Chiner E, Perelló S, Rubinós G, Mínguez O, Pascual L, Cortijo A, Martínez D, Aldomà A, Dalmases M, McEvoy RD, Barbé F. Effect of obstructive sleep apnoea and its treatment with continuous positive airway pressure on the prevalence of cardiovascular events in patients with acute coronary syndrome (ISAACC study): a randomised controlled trial. The Lancet Respiratory medicine 2020; 8: 359-367.

25. Eastwood PR, Barnes M, MacKay SG, Wheatley JR, Hillman DR, Nguyên XL, Lewis R, Campbell MC, Pételle B, Walsh JH, Jones AC, Palme CE, Bizon A, Meslier N, Bertolus C, Maddison KJ, Laccourreye L, Raux G, Denoncin K, Attali V, Gagnadoux F, Launois SH. Bilateral hypoglossal nerve stimulation for treatment of adult obstructive sleep apnoea. The European respiratory journal 2020; 55.

26. Peker Y, Glantz H, Eulenburg C, Wegscheider K, Herlitz J, Thunstrom E. Effect of Positive Airway Pressure on Cardiovascular Outcomes in Coronary Artery Disease Patients with Nonsleepy Obstructive Sleep Apnea. The RICCADSA Randomized Controlled Trial. American journal of respiratory and critical care medicine 2016; 194: 613-620.

27. Peker Y, Glantz H, Thunstrom E, Kallryd A, Herlitz J, Ejdeback J. Rationale and design of the Randomized Intervention with CPAP in Coronary Artery Disease and Sleep Apnoea-RICCADSA trial. Scand Cardiovasc J 2009; 43: 24-31.

28. Sleep-related breathing disorders in adults: recommendations for syndrome definition and measurement techniques in clinical research. The Report of an American Academy of Sleep Medicine Task Force. Sleep 1999; 22: 667-689.

29. Johns MW. A new method for measuring daytime sleepiness: the Epworth sleepiness scale. Sleep 1991; 14: 540-545.

30. Zung WW. A rating instrument for anxiety disorders. Psychosomatics 1971; 12: 371-379.

31. Dunstan DA, Scott N, Todd AK. Screening for anxiety and depression: reassessing the utility of the Zung scales. BMC Psychiatry 2017; 17: 329.

32. Obesity: preventing and managing the global epidemic. Report of a WHO consultation. World Health Organ Tech Rep Ser 2000; 894: i-xii, 1-253.

33. Sawyer AM, Gooneratne NS, Marcus CL, Ofer D, Richards KC, Weaver TE. A systematic review of CPAP adherence across age groups: clinical and empiric insights for developing CPAP adherence interventions. Sleep medicine reviews 2011; 15: 343-356.

34. Lederer DJ, Bell SC, Branson RD, Chalmers JD, Marshall R, Maslove DM, Ost DE, Punjabi NM, Schatz M, Smyth AR. Control of confounding and reporting of results in causal inference studies. Guidance for authors from editors of respiratory, sleep, and critical care journals. Annals of the American Thoracic Society 2019; 16: 22-28.

35. Grillon C. Models and mechanisms of anxiety: evidence from startle studies. Psychopharmacology (Berl) 2008; 199: 421-437.

36. Kustrzycki W, Rymaszewska J, Malcher K, Szczepanska-Gieracha J, Biecek P. Risk factors of depressive and anxiety symptoms 8 years after coronary artery bypass grafting. Eur J Cardiothorac Surg 2012; 41: 302-306.

37. Leal PC, Goes TC, da Silva LCF, Teixeira-Silva F. Trait vs. state anxiety in different threatening situations. Trends Psychiatry Psychother 2017; 39: 147-157. 
38. Grace SL, Abbey SE, Irvine J, Shnek ZM, Stewart DE. Prospective examination of anxiety persistence and its relationship to cardiac symptoms and recurrent cardiac events. Psychother Psychosom 2004; 73: 344-352.

39. Lett HS, Blumenthal JA, Babyak MA, Sherwood A, Strauman T, Robins C, Newman MF. Depression as a risk factor for coronary artery disease: evidence, mechanisms, and treatment. Psychosom Med 2004; 66: 305-315.

40. Reyes-Zuniga M, Castorena-Maldonado A, Carrillo-Alduenda JL, Perez-Padilla R, Martinez-Estrada A, Gomez-Torres L, Torre-Bouscoulet L. Anxiety and depression symptoms in patients with sleep-disordered breathing. Open Respir Med J 2012; 6: 97-103.

41. Lee SA, Paek JH, Han SH. REM-related sleep-disordered breathing is associated with depressive symptoms in men but not in women. Sleep \& breathing = Schlaf \& Atmung 2016; 20: 9951002.

42. Yue T, Li Q, Wang R, Liu Z, Guo M, Bai F, Zhang Z, Wang W, Cheng Y, Wang H. Comparison of Hospital Anxiety and Depression Scale (HADS) and Zung Self-Rating Anxiety/Depression Scale (SAS/SDS) in Evaluating Anxiety and Depression in Patients with Psoriatic Arthritis. Dermatology 2019: 1-9.

43. Luyster FS, Strollo PJ, Jr., Thunstrom E, Peker Y. Long-term use of continuous positive airway pressure therapy in coronary artery disease patients with nonsleepy obstructive sleep apnea. Clinical cardiology 2017; 40: 1297-1302.

44. Patil SP, Ayappa IA, Caples SM, Kimoff RJ, Patel SR, Harrod CG. Treatment of adult obstructive sleep apnea with positive airway pressure: an American Academy of Sleep Medicine systematic review, meta-analysis, and GRADE assessment. Journal of clinical sleep medicine: JCSM: official publication of the American Academy of Sleep Medicine 2019; 15: 301. 


\section{Figure legends}

Figure 1. Flow of patients through the study.

Definition of abbreviations: $\mathrm{AHI}=$ apnea-hypopnea index; $\mathrm{CAD}=$ coronary artery disease;

$\mathrm{CPAP}=$ continuous positive airway pressure; $\mathrm{CSA}-\mathrm{CSR}=$ central sleep apnea-Cheyne Stokes respiration; ESS $=$ Epworth Sleepiness Scale; OSA = obstructive sleep apnea; $\mathrm{RCT}=$ Randomized Controlled Trial; RICCADSA $=$ Randomized Intervention with CPAP in Coronary Artery Disease and Sleep Apnea.

Figure 2A. Distribution of delta scores on Zung Self-rated Anxiety Scale at 3- and 12-month follow-ups in intention-to-treat population.

Figure 2B. Distribution of delta scores on Zung Self-rated Anxiety Scale at 3- and 12-month follow-ups in on-treatment population.

Definition of abbreviations: $\mathrm{CPAP}=$ continuous positive airway pressure.

Figure 3. Correlation between accumulated CPAP hours/day and change on Zung Self-rated Anxiety Scale scores from baseline at 12-month follow-up in the entire study population.

Definition of abbreviations: $\mathrm{CPAP}=$ continuous positive airway pressure. 
Table 1. Baseline characteristics of the entire study population with coronary artery disease and nonsleepy obstructive sleep apnea $(n=208)$.

\begin{tabular}{lcc}
\hline & $\begin{array}{c}\text { CPAP } \\
(\mathbf{n}=\mathbf{1 0 3})\end{array}$ & $\begin{array}{c}\text { No-CPAP } \\
(\mathbf{n}=\mathbf{1 0 5})\end{array}$ \\
\hline Demographic characteristics & & \\
\hline Age, y & $65.9(60.5-70.8)$ & $67.4(61.0-73.1)$ \\
Age $\geq 65 \mathrm{y}, \%$ & 53.4 & 55.2 \\
BMI kg/m ${ }^{2}$ & $27.8(25.6-30.1)$ & $28.7(26.7-30.1)$ \\
Obesity, \% & 26.2 & 26.7 \\
Male sex, \% & 83.5 & 83.8 \\
\hline Home sleep apnea testing & & \\
\hline AHI, events/h & $23.9(17.9-36.8)$ & $24.8(18.8-35.4)$ \\
\hline Comorbidities & & \\
\hline Current smoking at baseline, $\%$ & 15.5 & 25.3 \\
CABG before inclusion, $\%$ & 26.2 & 48.6 \\
AMI at baseline, $\%$ & 55.3 & 17.1 \\
Former revascularization, $\%$ & 19.4 & 60.0 \\
Hypertension, $\%$ & 68.9 & $16.2 *$ \\
Diabetes mellitus, $\%$ & 28.2 & 6.7 \\
Stroke, $\%$ & 7.8 & 8.6 \\
Pulmonary disease, $\%$ & 3.9 & 1.9 \\
Anti-depressive medication, $\%$ & 4.0 & \\
\hline Questionnaires & & $5.0(4.0-7.0)$ \\
\hline ESS score & $6.0(4.0-8.0)$ & 26.0 \\
Zung SDS score $\geq 50, \%$ & 29.0 & $37.5(32.5-43.8)$ \\
Zung SAS score & $37.5(32.5-41.3)$ & 21.9 \\
Anxiety (Zung SAS score $\geq 45), \%$ & 13.6 &
\end{tabular}

Continuous variables are expressed as median and bounderies of interquartile ranges.

Definitions of abbreviations: AMI, acute myocardial infarction; AHI, apnea hypopnea index; BMI, body mass index; $\mathrm{CABG}$, coronary artery bypass graft; $\mathrm{CPAP}$, continuous positive airway pressure; ESS, epworth sleepiness scale; PCI, percutaneous coronary intervention; SAS, self-rating anxiety scale; SDS, self-rating depression Scale.

$* p<0.05$ 
Table 2. Between-group comparisons of the absolute values of the Zung SAS scores in the entire study cohort at baseline and follow-ups.

\begin{tabular}{|c|c|c|c|c|}
\hline & \multicolumn{2}{|r|}{ Baseline } & \multirow{2}{*}{$\begin{array}{c}\text { After 3 Months } \\
\begin{array}{c}\text { Median } \\
\left(25^{\text {th }_{0}} \%-75^{\text {th }_{0}} \%\right)\end{array} \\
\end{array}$} & \multirow{2}{*}{$\begin{array}{c}\text { After 12 Months } \\
\begin{array}{c}\text { Median } \\
\left(25^{\text {th }_{0}} \%-75^{\text {th }_{0}} \%\right)\end{array}\end{array}$} \\
\hline & $\mathrm{n}$ & $\begin{array}{c}\text { Median } \\
\left(25^{\text {th}_{0}} \%-75^{\text {th }} \%\right)\end{array}$ & & \\
\hline $\begin{array}{l}\text { Intention-to-treat } \\
\text { population } \\
\text { CPAP }\end{array}$ & 103 & $37.5(32.5-41.3)$ & $36.9(31.3-41.3)$ & $36.3(31.3-40.0)$ \\
\hline No-CPAP & 105 & $37.5(32.5-43.8)$ & $38.2(32.5-42.5)$ & $36.3(31.3-42.5)$ \\
\hline & & n.s. & n.s. & n.s. \\
\hline $\begin{array}{l}\text { On-treatment } \\
\text { population } \\
\text { CPAP usage } \geq 2.8 \\
\text { hours/day }\end{array}$ & 59 & $36.3(31.3-41.3)$ & $37.5(31.3-41.3)$ & $36.3(30.0-42.5)$ \\
\hline $\begin{array}{l}\text { CPAP usage }<2.8 \\
\text { hours/day or no-CPAP }\end{array}$ & 149 & $\begin{array}{c}38.8(33.8-43.8) \\
p=0.009\end{array}$ & $\begin{array}{c}37.5(32.5-42.5) \\
\text { n.s. }\end{array}$ & $\begin{array}{c}36.3(31.3-41.3) \\
\text { n.s. }\end{array}$ \\
\hline
\end{tabular}

Definitions of abbreviations: CPAP, continuous positive airway pressure; SAS, self-rated anxiety scale. P-values refer to between-group differences in absolute values of the SAS scores. 
Table 3. Predictors of nonadherence among patients randomized to CPAP at baseline ( $n=103)$.

\begin{tabular}{lccc}
\hline Multivariate & Odds Ratio & $\mathbf{9 5 \%}$ CI & $\boldsymbol{p}$-values \\
\hline Age & 1.00 & $0.95,1.05$ & 0.912 \\
Male sex & 1.09 & $0.35,3.46$ & 0.879 \\
CABG at baseline & 0.83 & $0.32,2.16$ & 0.832 \\
ESS score at baseline (0-9) & 0.99 & $0.82,1.19$ & 0.933 \\
Zung SAS score at baseline & $\mathbf{1 . 1 1}$ & $\mathbf{1 . 0 4 , \mathbf { 1 . 1 8 }}$ & $\mathbf{0 . 0 0 3}$ \\
\hline
\end{tabular}

Definition of the abbreviations: CABG, coronary artery bypass grafting; CI, confidence interval; CPAP, continuous positive airway pressure; ESS, epworth sleepiness scale; SAS, self-rated anxiety scale. 
Table 4. Predictors of change from baseline in SAS scores at 12- month-follow-up in the entire study population $(\mathrm{n}=208)$.

\begin{tabular}{lccc}
\hline Multivariate & Standardized $\boldsymbol{\beta}$ & $\mathbf{9 5 \%}$ CI & $\boldsymbol{p}$-values \\
\hline Age & 0.001 & $-0.108,0.107$ & 0.990 \\
Male sex & -0.096 & $-3.893,0.748$ & 0.183 \\
CABG at baseline & -0.077 & $-3.139,0.984$ & 0.304 \\
AMI at baseline & -0.066 & $-2.602,0.981$ & 0.373 \\
Pulmonary disease & 0.023 & $-3.031,4.168$ & 0.756 \\
Accumulated CPAP hours/day & $\mathbf{0 . 1 4 4}$ & $\mathbf{0 . 0 0 5 , 0 . 6 9 5}$ & $\mathbf{0 . 0 4 7}$
\end{tabular}

Definition of the abbreviations: AMI, acute myocardial infarction; CABG, coronary artery bypass grafting; $\mathrm{CI}$, confidence interval; CPAP, continuous positive airway pressure; SAS, self-rated anxiety scale. 
1291 revascularized CAD patients were assessed for eligibility

629 were excluded:

32 had known OSA

597 were not interested in the study

662 underwent home sleep apnea testing and answered the ESS questionnaire

\begin{tabular}{|c|c|}
\hline & $\begin{array}{l}151 \text { were excluded: } \\
101 \text { had borderline OSA (AHI 5.0-14.9 } \mathrm{h}^{-1} \text { ) } \\
21 \text { had predominant CSA/CSR } \\
29 \text { declined further investigations }\end{array}$ \\
\hline \multicolumn{2}{|c|}{511 met the inclusion criteria for the main RICCADSA trial } \\
\hline & $\begin{array}{l}267 \text { were excluded from the RCT } \\
112 \text { had no OSA }\left(\mathrm{AHI}<5 \mathrm{~h}^{-1}\right) \\
155 \text { had Sleepy OSA }\left(\mathrm{AHI} \geq 15 \mathrm{~h}^{-1} ; \mathrm{ESS} \geq 10\right)\end{array}$ \\
\hline \multicolumn{2}{|c|}{$\begin{array}{c}244 \text { had Nonsleepy OSA (AHI } \geq 15 h^{-1} ; \mathrm{ESS}<10 \\
\text { Included in the RCT } \text { arm }\end{array}$} \\
\hline 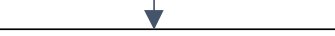 & $\nabla$ \\
\hline $\begin{array}{l}\text { Assigned to CPAP } \\
\qquad(\mathrm{n}=122)\end{array}$ & $\begin{array}{l}\text { Assigned to no-CPAP } \\
\qquad(\mathrm{n}=122)\end{array}$ \\
\hline
\end{tabular}

28 were excluded due to no or incomplete Zung SAS questionnaires at baseline and/or at follow-ups

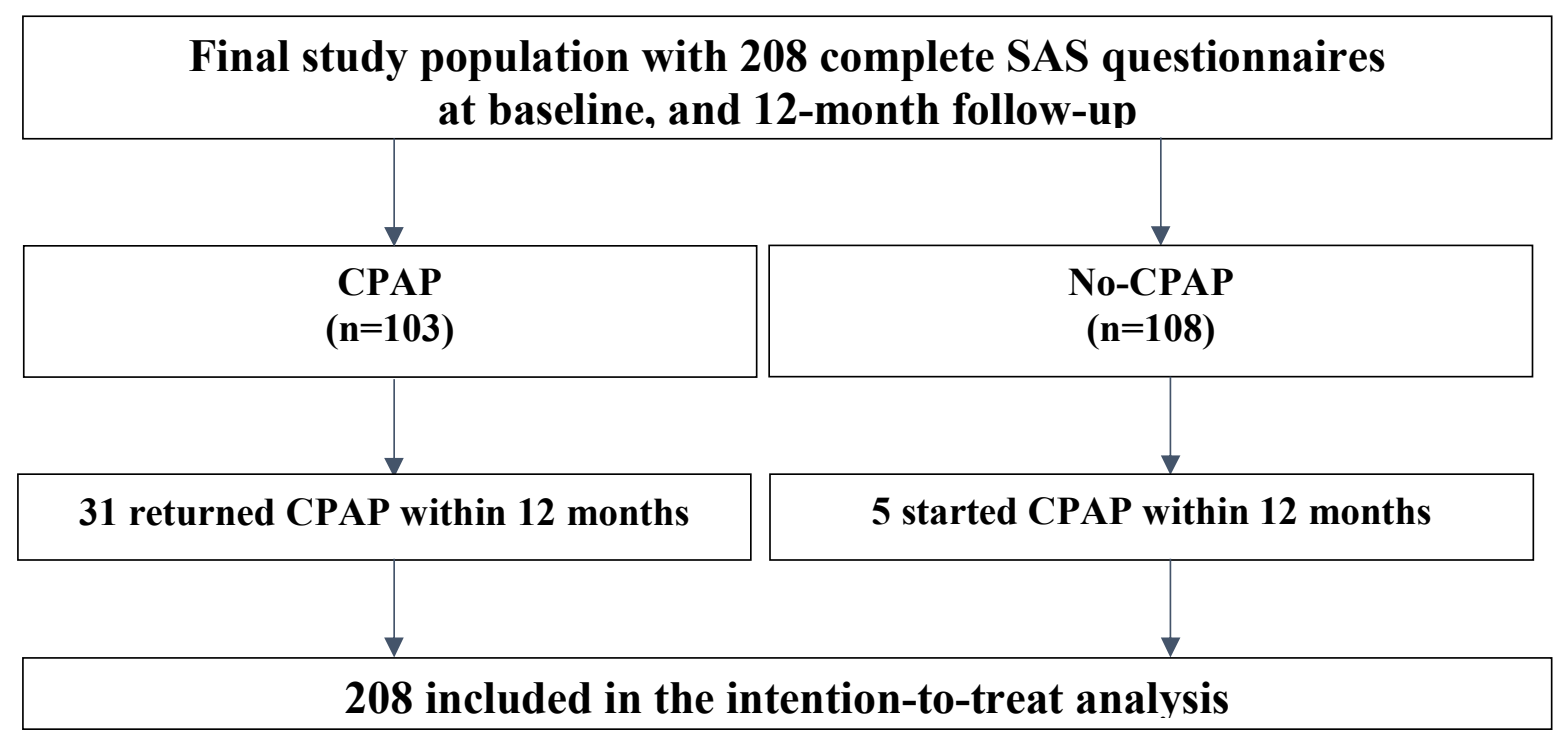




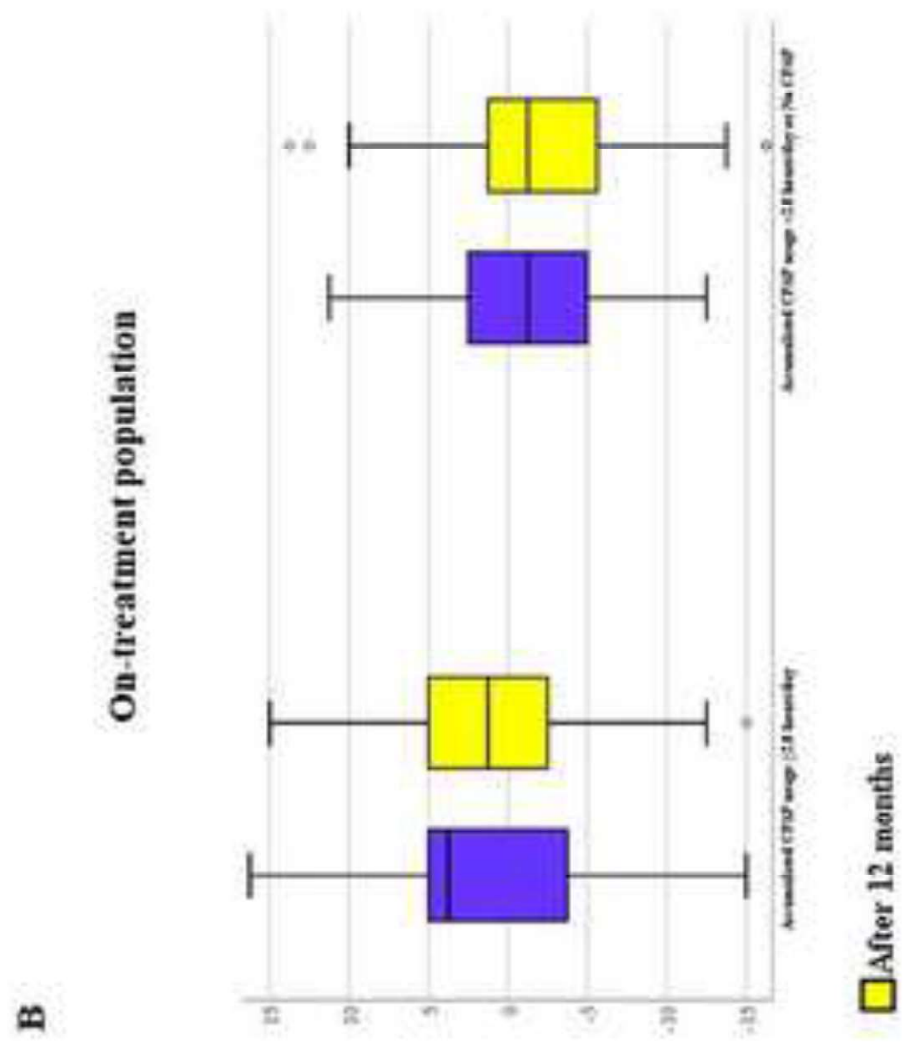

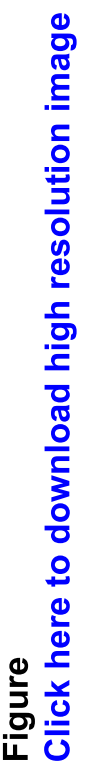

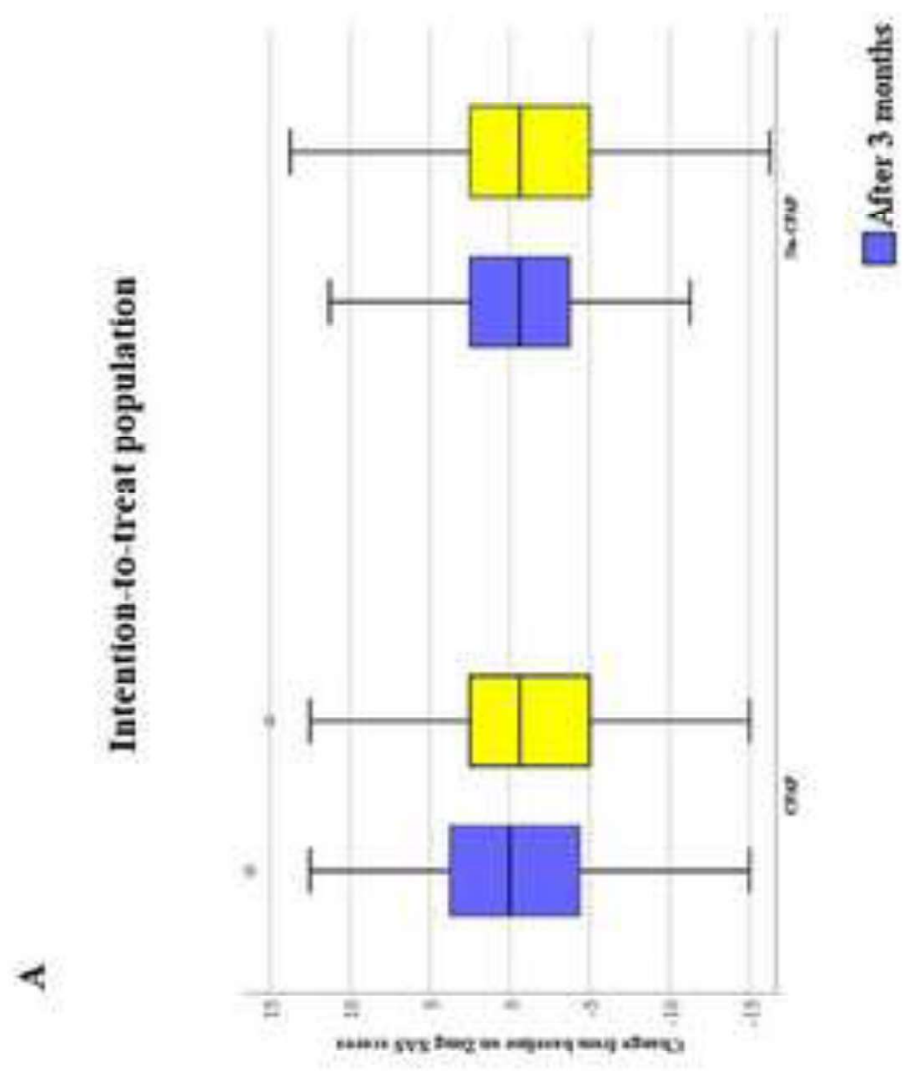




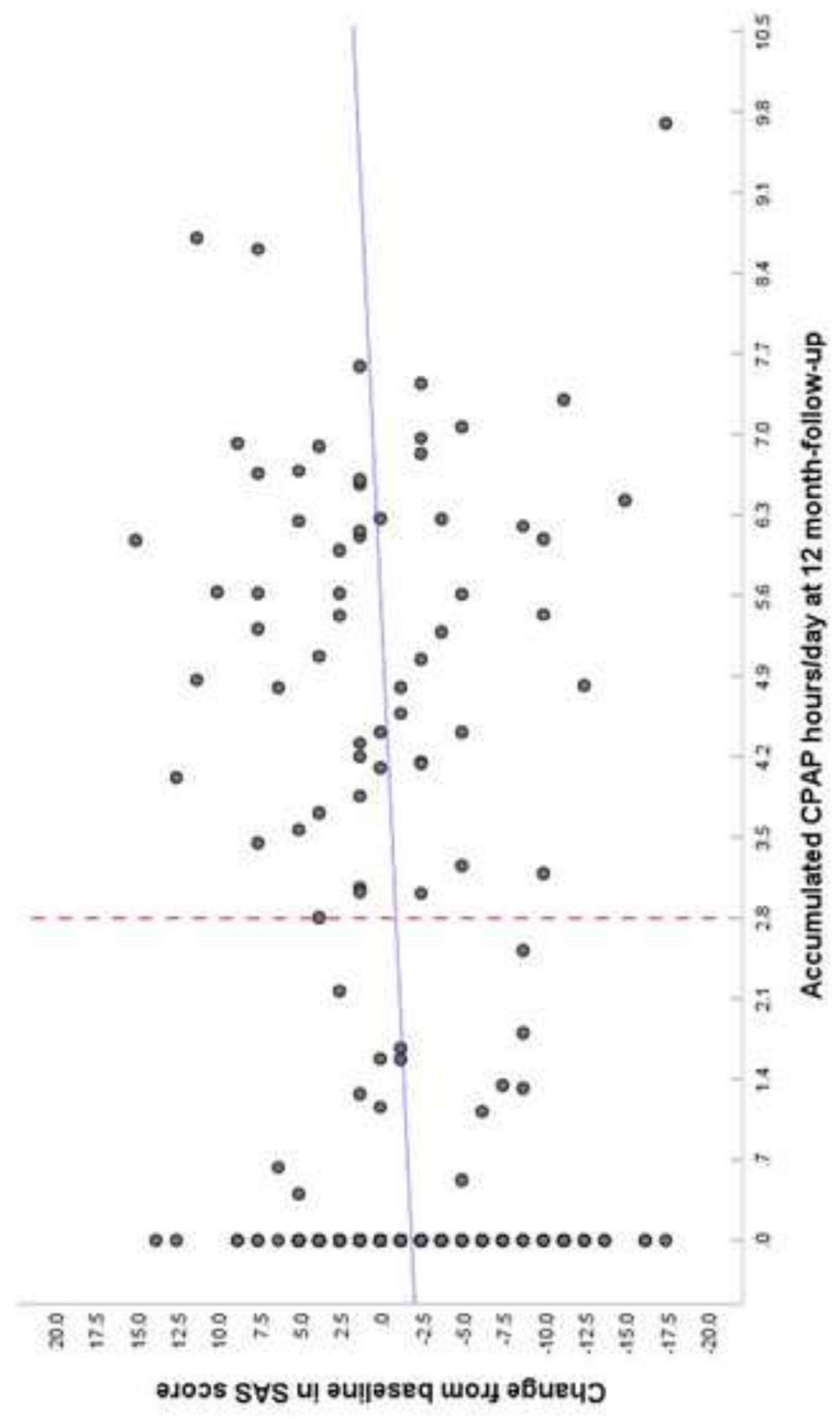

